\title{
Accounting
}

\section{Crypto-currency: Empirical evidence from GSADF and wavelet coherence techniques}

\author{
Dervis Kırıkkalelia $^{*}$, Ersin Cağlar ${ }^{a}$ and Kelvin Onyibor ${ }^{*}$
}

${ }^{a}$ Department of Banking and Finance, Faculty of Economic and Administrative Science, European University of Lefke, Northern Cyprus, TR-10 Mersin, Turkey

${ }^{b}$ Department of Management Information Systems, European University of Lefke, Northern Cyprus, TR-10 Mersin, Turkey

${ }^{c}$ Department of Economics, University of Manitoba, Canada

CHRONICLE ABSTRACT

Article history:

Received September 22019

Received in revised format

September 22019

Accepted October 152019

Available online

October 172019

Keywords:

Crypto-currency

Multiple Bubble

GSADF

Wavelet Coherence

\begin{abstract}
This study is targeted towards the explosive behavior of crypto-currencies, namely Bitcoin, Etherium, Litecoin and Ripple by investigating the crypto-currencies bubbles and the causal link between Bitcoin and other three crypto-currencies prices, using GSADF and wavelet coherence tests. The study aims to answer the following questions which have not been investigated in the literature to our best knowledge (i) Was there any bubble in the prices of Bitcoin, Etherium, Litecoin and Ripple and between 01.09.2016 and 01.04.2019? If yes, why (ii) was there any linkage between Bitcoin and Etherium, Litecoin and Ripple? Our findings reveal that (a) there were some bubbles in the cryptocurrencies for the periods investigated; (b) there was a positive correlation between Bitcoin and Etherium, Litecoin and Ripple in the short-run; (c) changes in Bitcoin prices lead changes Etherium, Litecoin and Ripple prices in the long run at different periods.
\end{abstract}

\section{Introduction}

Crypto-currency was first introduced into the financial market mid-way into the year 2000. Its ever-rising popularity has spread across the globe like a wide fire as a result of its decentralized nature in the monetary and banking systems, hence, standing as a competitive threat against the already existing traditional centralized financial, banking and monetary systems. However, no studies have venture to draw evidence of the presence of explosivity (bubble) in major present leading crypto-currencies which now constitute a large share of the market capitalization. Hence, this is the gap our study seeks to fill in the literature, employing both single and multiple bubble period detection techniques of right tailed ADF; Standard ADF (Dickey, 1984), Supremum ADF (Phillips et al., 2011; Phillips \& Yu, 2012), Backward ADF (Phillips et al., 2012), and Generalized Supremum ADF (Phillips et al., 2015) test and also the time and frequency domain techniques (wavelet coherence) on the 4 leading cryptocurrencies (Bitcoin, Ethereum, Litecoin and Ripple) to detect price bubble in the crypto-currency market and presence of causal linkage among these electronic coins.

The creation of this electronic currency emanated as a corrective measure in an attempt to pretend the re-occurrence of the 2008 Global Financial Crisis and the failure of the United States financial and monetary systems (Bariviera et al., 2017). The number

* Corresponding author. Tel.: +90 5488637770

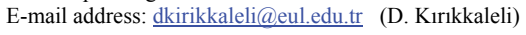


of crypto-currencies has vastly increased geometrically over the years, currently there are around a thousand functioning cryptocurrency in the coin market today which exist and function in various forms. These currencies have posed a challenge against Fiat currencies by presenting the world with a flexible system of payment which is absolutely independent from the control of the instituted financial authorities, Hence, eliminating the role of third party in payment process and currencies exchange. Having attained a plausible $\$ 70.8$ billion market capitalization (Cap, 2018), Bitcoin has been considered as the most popular crypto-currency in the coin market, however the growth in popularity of other existing crypto-currencies stands as a major interest and concern in the electronic money market. It is worthy to point out that, Etherium and Ripple, having achieved a market cap of $\$ 15.9$ billion and $\$ 15.1$ billion respectively have been considered as the major rival to Bitcoin. The increasing popularity in these alt coins are gradually crippling Bitcoin market share. However, Bitcoin still holds a resounding $45.7 \%$ of total estimated market capitalization in the coin market (Cap, 2018).

The ever-rising prices of these crypto-currencies have drawn much attention to the market, for instance, Bitcoin rising has grown by $122 \%$ in 2016 and $1360 \%$ in 2017. According to Fry and Cheah (2016) Bitcoin holds the position of the highest price bubble recorded in a financial asset despite its plausible independence in operations. Recent research on this field (Chueng et al., 2015; Fry \& Cheah, 2016; Corbet et al., 2017; Su et al., 2018) have directed its attention in presenting empirical results of the presence of bubble in these crypto-currencies and the presence of a co-explosive behavior among them. According to Semiromi and Reza (2010), bubbles are economic and financial phenomena which are observable. Speculative (asset price) bubble is a term that explains a persistent market situation of overvaluation in asset which is often preceded by a collapse in the market. The presence of bubble can be made eminent when an asset's fundamental values is consistently below its market selling price and the fundamental value consists of both the future selling price and its aggregate future dividend during the holding period. Hence, an asset price bubble is considered as the difference between the fundamental value of an asset and its corresponding market selling price (Semiromi \& Reza, 2010). According to Larsen (1997), a price bubble occurs when an initial increase in an asset's value leads to a further increase in its current market value. This would suggest that traders (investors) are most likely going to attach more value to an asset if its market price is on a persistent rise and this would increase the desire to acquire and hold these assets.

For the course of this study, attention would be greatly drawn to 2 important studies. Bouri et al. (2018) is believed to be the first who investigated the presence of a co-explosivity in the crypto-currency market, drawing evidence from Bitcoin. Second Corbet et al. (2017), showed the presence of bubble in the Ethereum market by employing the Supremum ADF test (Philips et al., 2011). In subsequent sections, the existing literature, methodology employed, results attained, conclusions drawn and recommendations given would be outlined respectively.

\section{Literature review}

Empirical evidence presented by Dyhrberg (2016) critically analyzes the statistical features of the crypto-currency market. In their study, they discovered that crypto-currencies do not meet the laid standards of a tradition tradable currency as suggested by the ECB (2012), since it's function as a medium of exchange, can barely be observed and finally the high level of price volatility of these digital coins could not allow it to function effectively as a store of value.

In an attempt to better understand the dynamics of the crypto-currency market, Dyhrberg (2016), analyzed both the short and long term past values of selected coins prices, (Bitcoin, Litcoin, Ethereum). The main aim of these study was to determine whether there exists an autocorrelation between current coin prices and the its past prices, i.e. whether the current price of a digital currency is being affected by the initial prices of the currency by employing hourly prices data in the analysis. Their results show a high correlation between past and future crypto-currency prices; however, a more extensive study was recommended in order to better understand the dynamics of the crypto-currency market. Further attempts to elaborate on this growing field were carried out by Bariviera et al. (2017) who tried to understand the position of crypto-currencies in the financial asset market and draw a linkage between cryptocurrencies and portfolio and risk management. By employing GARCH model, Dyhrberg (2016) tried to create a comparative scenario with reference to correlation of correlation between crypto-currencies with both US dollar and Gold. As a result of the limited scope in data range used in this study, (2013-2015). The true characteristics of the crypto-currency market was not captured in her analysis, reason being that the main volatility in price behavior of these digital currencies are more concentrated around 2016 to 2017 and as such, this created a need for further studies in these field.

However, Baur et al. (2018), generated some empirical criticism against the earlier study of Dyhrberg (2016) who employed strict econometric methodology. In their study they took further steps in extending the data horizon and adopted a rather descriptive statistics, since according to them, the factors that drive fluctuations in Bitcoin are more socially inclined. Finally, they concluded that no correlation exists between crypto-currencies with portfolio and risk management since the former those not exhibit the characteristics of fiat currencies. 
Several existing studies have tried to evaluate Bitcoin returns statistical components. The Bitcoin time vary volatility model was structured by Pichl and Kaizoji (2017) and a relatively higher significance was discovered compared to fiat currencies. A further attempt was made by Urquhart and Zhang (2018) employing several volatility GARCH models and statistically investigating the crypto-currency's hedging potentials against its rival currencies. The study of the hedging potential was earlier investigated by Bouri et al. (2017) using a Hurst exponent analysis of vast memory to generate results for clustering volatility. In the same vein, Osterrieder and Lorenz (2017) discovered an accurate prediction of the size of the crashes that occurs in the Bitcoin prices using order book data of Bitcoin liquidity base.

On the other hand, there exists non-statistical modeling and findings of the crypto-currency price market. Fantazzini et al. (2016) outlined the dynamics surrounding the social component of the crypto-currency market using Bitcoins as a singular case study for its analysis. In addition, Kristoufek (2013) established an association between Bitcoins and internet search queries. \

The above-discussed findings of the crypto-currencies price market and its related social dynamics has strongly suggested that users of these digital coins have been attracted by its ever-rising price trend and simultaneously been influenced by social media and related news. This market behavior is typical of financial related bubbles as seen in other price bubble like the stock market price bubble, Bernanke, B. S. (2002). Hence, in this paper, we would be employing strong statistical and financial modeling to detect whether the crypto-currency market shows a high speculative attribute that is, evaluating its explosivity (bubble) trend.

\section{Methodology}

This study seeks to examine the micro dynamic relationships that exist among the major crypto-currencies. In this research, daily coin prices of the selected crypto-currencies from 01.09.2016 to 19.03.2019 were used. Data time frame was selected based on observed significant fluctuation period of coin prices, since earlier coin price trends exhibit a rather linear movement. Dataset was collected from the official Coinbase statistical webpage, (Coinbase, 2018). Firstly, in order to examine the existence of a price bubble in the Crypto-currency market, the Generalized Suprenum Augmented Dickey Fuller test analysis was employed. Phillips et al. (2015) developed the GSADF, which is a re-modification of the Sup ADF test. The GSADF test is designed to capture multiple bubbles in an asset price, which in this context would be the coin prices of the 4 highest traded crypto-currencies. As demonstrated by Phillips et al. (2015), the GSADF outperform the sup ADF test statistics as the latter only detect single price bubble in a given time series data. In addition, the GSADF gives an opportunity to manually determine the window width as well as the start and endpoint in its estimations. PSY test allows for flexibility whereby the regression starting point $\left(r_{1}\right)$ changes within a range which is from 0 to $\left(r_{2}-r_{0}\right)$ while the regression endpoint $\left(r_{2}\right)$ also changes from the minimal window width $\left(\mathrm{r}_{0}\right)$ to 1.Implementation of the GADF is shown graphically in Fig. 1. Eq. (1) defines the GSADF test statistic as:

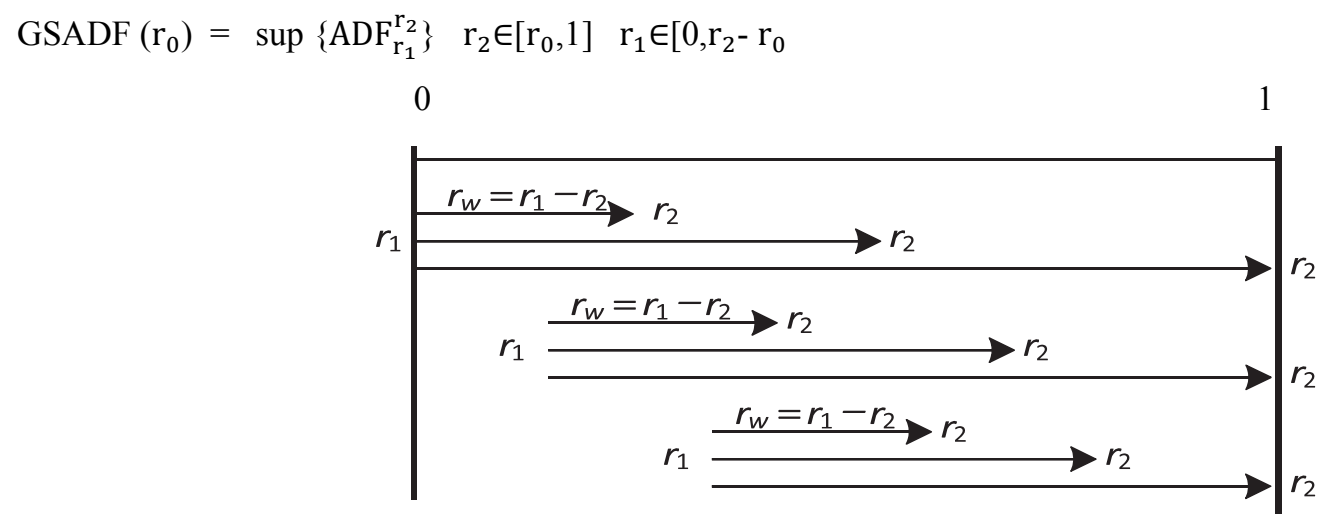

Fig. 1. GSADF implementation

The null hypothesis for the data generating process (Eq. 1) was set as $Q=1$ (No price bubble) against the alternative $\varrho>1$ (Price bubble) by Phillips et al. (2015) to investigate the price explosivity behavior. Hence implementing Eq. (1) into our study model gives;

$$
\begin{aligned}
& B T C_{t}=d \mathrm{~S}^{-\mathrm{j}}+\varrho B B T C_{t-1}+e_{t} \\
& L T C_{t}=d \mathrm{~K}^{-\mathrm{j}}+\varrho L T C_{t-1}+e_{t} \\
& E T H_{t}=d \mathrm{~W}^{-\mathrm{j}}+\varrho E T H_{t-1}+e_{t} \\
& X R P_{t}=d \mathrm{~L}^{-\mathrm{j}}+\varrho X R P_{t-1}+e_{t}
\end{aligned}
$$


where BTC, LTC, ETH and XRP represent the 4 crypto-currencies (Bitcoin, Litcoin, Etherium and Ripple respectively) employed in this study, $\mathrm{S}, \mathrm{K}, \mathrm{W}$ and $\mathrm{L}$ are the stated sample sizes, $d$ and $j$ are constants with $\mathrm{j}>0.5$. Both the drift and constant size are controlled by $j$ while $S, K, W$ and $L \rightarrow \infty$. According to Corbet et al. (2017), as long as $j \geq 0.5$ the distribution of ADF would not be affect by fluctuations. Hence, we follow the recommendation of Phillips et al. (2015) by setting $d$ and $j$ to equal 1 . For the course of this study, $k_{0}=0.1$ is taken as the estimated window with 23 observations. The $k_{1}$ th of the entire sample size is considered as the initial points of both the flexible and rolling window. While its end point is defined as $k_{2} t h^{4}$. Hence, Eqs. (1-4) can be re-written as:

$$
\begin{aligned}
& \Delta B T C_{t}=\boldsymbol{r}_{k 1, k 2}+\mathfrak{b}_{k 1, k 2} B T C_{t-1}+\sum_{i=1}^{m} \mathrm{U}_{k 1, k 2}^{i} \Delta B T C_{t-1}+e_{t}, \\
& \Delta L T C_{t}=\boldsymbol{r}_{k l, k 2}+\mathfrak{b}_{k 1, k 2} L T C_{t-1}+\sum_{i=1}^{m} \mathrm{U}_{k 1, k 2}^{i} \Delta L T C_{t-1}+e_{t,} \\
& \Delta E T H_{t}=\boldsymbol{r}_{k 1, k 2}+\mathfrak{b}_{k 1, k 2} E T H_{t-1}+\sum_{i=1}^{m} \mathrm{U}_{k 1, k 2}^{i} \Delta E T H_{t-1+} e_{t,} \\
& \Delta X R P_{t}=\boldsymbol{r}_{k l, k 2}+\mathfrak{b}_{k l, k 2} X R P_{t-1}+\sum_{i=1}^{m} \mathrm{U}_{k 1, k 2}^{i} \Delta X R P_{t-1+}+e_{t .}
\end{aligned}
$$

In the above estimated models, $T_{w}=k_{w} T$ is the sample size, $m$ is the selected lag length according to the SIC lag selection test. As a further step after price bubble has been detected through the GSADF test, we go on to date stamp the start and end point of the bubble periods by employing the backward SADF test developed by Phillips et al. (2015):

$$
\operatorname{BSADF}_{r 2}\left(r_{0}\right)=\operatorname{Sup}_{r 1 \in(0, r 2-r 1)} A D F_{r 1}^{r 2}
$$

where; $r_{2}$ denotes the fixed window of the rolling interval and the window size ranges from $r_{0}$ to $r_{2}$. The periods of price explosivity is set by the GSADF test as follows:

$$
\begin{aligned}
& \hat{r}_{e}=\inf _{r 2 \in(r 0,1)}\left\{r_{2}: B S A D F_{r 2}>c v_{r 2}^{\alpha T},\right. \\
& \hat{r}_{f}=\inf _{r 2 \in\left(\hat{r}_{e}, 1\right)}\left\{r_{2}: B S A D F_{r 2}>c v_{r 2}^{\alpha T},\right.
\end{aligned}
$$

where is $c v \frac{\alpha T}{r 2}$ represents the $5 \%$ critical value of SADF statistics based on $\mathrm{r} 2$ observations, and $\alpha_{t}$ is a constant value of $5 \%$. According to Astill et al. (2017), for investigating the existence of a potential non-stationary volatility, a wide bootstrapping technique is been employed. $R_{0}=0.01+1.8 / \sqrt{T}$ denotes the initial sample size (Philips et al., 2015). 1000 replications were selected for all simulations. As a major step in creating a gap in the crypto-currency market, we took a step further to examine the co-explosivity behavior among the selected coins in order to determine whether there is any form of correlation among the price behavior or on the other hand whether crypto-coins prices fluctuations are independent from each other using Bitcoin primary base for the estimation since it holds approximately $50 \%$ of the entire market capitalization. Hence, the wavelet coherence technique, developed by Goupillaud et al. (1984) would be employed to measure the time-frequency dependence of Bitcoin and its major rival crypto-currencies. In most economic and financial modeling both time and frequency-based techniques have been employed independently to investigate causal relationship that exist between economic and financial indicators. This approach often provides a spurious and unreliable results since financial and economic variables are nonstationary. Furthermore, according to Pal and Mitra (2017), fixed parameter traditional time-based causality test results tends to under conditions where there exist structural break(s) in the time series variables. On the other hand, Pal and Mitra, (2017) empirically proved that singular frequency base technique, also called Fourier transform, faces a major shortcoming since it fails to take into consideration the time base information of the variables. Hence, this study seeks to explore the dynamic behavior of the crypto-coins using the wavelet coherence technique which inculcate both the time and frequency component of the financial variables. The wavelet coherence technique falls under the Morlet wavelet family. Estimated below is the wavelet equation;

$$
\mathfrak{w}(t)=\pi^{-\frac{1}{4}} e^{-i \omega_{0} t} e^{-\frac{1}{2} t^{2}}, \mathrm{p}(\mathrm{t}), \mathrm{t}=1,2,3 \ldots ., \mathrm{T},
$$

where; the number of observations under the wavelet analysis is defined. Briefly, we can state that, the 2 parameters associated with the wavelet include the frequency (f) and time (k). The major function of the frequency parameter in the wavelet model is to control the expanded wavelet for restricting various frequencies while the time parameter describes a wavelet's actual position in time through wavelet exchange. Transforming $w$ would result to $\mathfrak{w}_{\mathrm{k}, \mathrm{f}}$. Hence the equation becomes:

$$
\mathfrak{w}_{k, f}(t)=\frac{1}{\sqrt{h}} w\left(\frac{t-k}{f}\right), \quad k, f \in \mathbb{R}, f \neq 0
$$

Furthermore, given the time series data $\mathrm{p}(\mathrm{t})$, the continuous wavelet is constructed from $\mathfrak{w}$ as a function of $k$ and $f$ as follows: 


$$
W_{p}(k, f)=\int_{-\infty}^{\infty} p(t) \frac{1}{\sqrt{f}} \psi\left(\frac{\overline{t-k}}{f}\right) d t .
$$

Eq. (16) shows the regenerated initial time series $\mathrm{p}(\mathrm{t})$ having a coefficient of $\mathfrak{w}$

$$
p(t)=\frac{1}{C_{\psi}} \int_{0}^{\infty}\left[\int_{-\infty}^{\infty}\left|W_{p}(a, b)\right|^{2} d a\right] \frac{d b}{b^{2}} .
$$

To statistically investigate the possibility of volatility in the time series variables at various time $(k)$ and frequencies $(f)$, the wavelet power spectrum is been employed as follows;

$$
W P S_{p}(k, f)=\left|W_{p}(k, f)\right|^{2}
$$

The main innovation of the wavelet coherence technique relative to the standard correlation is that the technique brings out any correlation between two time series $\mathrm{p}(\mathrm{t})$ and $\mathrm{q}(\mathrm{t})$ throughout the selected time period in combined time-frequency based causalities. The major advancement the wavelet coherence technique holds over standard traditional correlation techniques is the fact that, it evaluates any existing correlation between two independent variables $\mathrm{p}(\mathrm{t})$ and $\mathrm{d}(\mathrm{t})$ at a range of selected time periods over varying levels of frequencies simultaneously. As shown in Eq. (7), Eq. (18) is the time series cross wavelet transform (CWT):

$$
W_{p q}(k, f)=W_{p}(k, f) \overline{W_{q}(k, f)}
$$

where both $W_{p}(k, f)$ and $W_{q}(k, f)$ show the cross wavelet transformation of $p(t)$ and $d(t)$ respectively, (Torrence \& Compo, 1998). The squared wavelet coherence equation is estimated as follows:

$$
R^{2}(k, f)=\frac{\left|C\left(f^{-1} W_{p q}(k, f)\right)\right|^{2}}{C\left(f^{-1}\left|W_{p}(k, f)\right|^{2}\right) C\left(f^{-1}\left|W_{q}(k, f)\right|^{2}\right)}
$$

In Eq. (19), $C$ denotes time which serves as a smoothing process over time, where $0 \leq \mathrm{R}^{2}(k, f) \leq 1$. If $\mathrm{R}^{2}(k, f)$ approaches 1 , this interpret as variables being correlated at a certain scale, filled in red color and with a black outline. On the other hand, if $\mathrm{R}^{2}(k, f)$ approaches 0 , then there exists no little or no significant correlation between the time series variables and is shown with in blue color. Lastly, the shorting coming of the $\mathrm{R}^{2}(k, f)$ which is the inability to provide information on directional sign of the correlation was tackle in the literature by Torrence and Compo (1998) who developed a strategy of investigating the differences in the wavelet coherence using deferrals in the wavering of 2 time series. Eq. (9) shows the difference phase wavelet coherence:

$$
\phi_{p q}(k, f)=\tan ^{-1}\left(\frac{\mathrm{L}\left\{C\left(f^{-1} W_{p q}(k, f)\right)\right\}}{o\left\{C\left(f^{-1} W_{p q}(k, f)\right)\right\}}\right),
$$

where, $\mathrm{L}$ and $O$ indicate an imaginary operator and a real part operator, respectively.

\section{Empirical Findings}

The study aim is to empirically explore the existence of price explosively and correlation that exists among the leading cryptocurrencies (namely, Bitcoin, Etherium, Litecoin, and Ripple). The Generalized SADF which outperforms the SADF, was employed to ascertain the possible existence of price bubble(s) among these crypto-currencies. Furthermore, the wavelet coherence between Bitcoin (top leading cryto-coin with over 70\% of market share) and the other top leading crypto-currencies (Etherium, Litecoin, and Ripple) was verified in an attempt to see whether price changes in Bitcoins causes changes in the prices of the above listed coins. Hence, investigating the level of independence among crypto-currencies in the short. Medium and long run. In this empirical analysis, weekly data of coin prices was used which ranges from the first week of 2016 to the last week of 2018, (Cap, 2018).

\subsection{Bubble(s) Detection using General Supreme ADF Test (GSADF)}

In recent time series analysis, price bubbles are often detected by employing the Generalized SADF methodology proposed by Phillip et al. (2011). The GSADF test which has been statistically proven to have outperformed the SADF (Salhi \& Alflayyeh, 2016) was used to test price behavior among Bitcoin, Etherium, Litecoin, and Ripple. The test statistics and corresponding 
critical values for the GSADF tests was obtained from Monte Carlo simulation with 1000 replications and are shown below in Table 1. In the test specification 40 observations was used as the window size when calculating the GSADF t-stat and the critical values.

Table 1

GSADF test

\begin{tabular}{|c|c|c|c|c|c|c|c|c|}
\hline \multirow{3}{*}{ t-cr. } & \multicolumn{2}{|c|}{ BTC } & \multicolumn{2}{|c|}{ ETHERIUM } & \multicolumn{2}{|c|}{ LITECOIN } & \multicolumn{2}{|c|}{ RIPPLE } \\
\hline & t-Stat & Prob. & t-Stat & Prob. & t-Stat & Prob. & t-Stat & Prob. \\
\hline & 5.505 & $0.000 *$ & 6.497 & $0.000 *$ & 5.804 & $0.000 *$ & 10.665 & $0.000^{*}$ \\
\hline $99 \%$ level & 1.791 & & 2.879 & & 1.791 & & 1.791 & \\
\hline $95 \%$ level & 1.299 & & 2.167 & & 1.299 & & 1.299 & \\
\hline $90 \%$ level & 1.172 & & 2.069 & & 1.172 & & 1.172 & \\
\hline
\end{tabular}

Notes: * indicate statistical significance at the $1 \%$. T-cr indicates test critical values which are based on a Monte Carlo simulation.

In Table 1, the estimated GSADF t-stat of Bitcoin is $5.505(0.000)$, which is greater than the corresponding $1 \%$, 5\%, 10\% critical values. This outcome shows that the null hypothesis of a random walk is rejected at all significant levels, hence, proving that Bitcoin exhibits an explosive behavior in its price trend. Furthermore, the GSADF Date-stamping strategy test result below shows that the bubble period for bitcoin exist from the 3rd quarter of 2016 to 4th quarter of 2016, 2nd quarter of 2017 to 3 rd quarter of 2017, from the 4th quarter of 2017 to 1st quarter of 2018. In the case of Etherium, the t-stat of the logarithm is 6.497 , which is much greater than the corresponding $1 \%, 5 \%, 10 \%$ critical values. Hence, this finding suggests that price bubble(s) exist in the Etherium market. This finding reflects the volatility of Etherium as shown by the result of the Date-stamping result below. As shown in the result bubbles exist between from the 2016, (2017Q1 to 2017Q3), and (2017Q4 to 2018Q2).

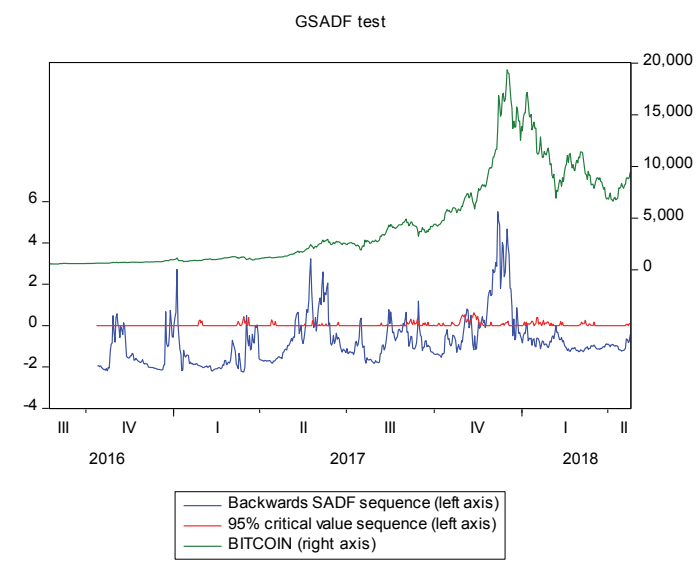

Fig. 1. GSADF test for Bitcoin

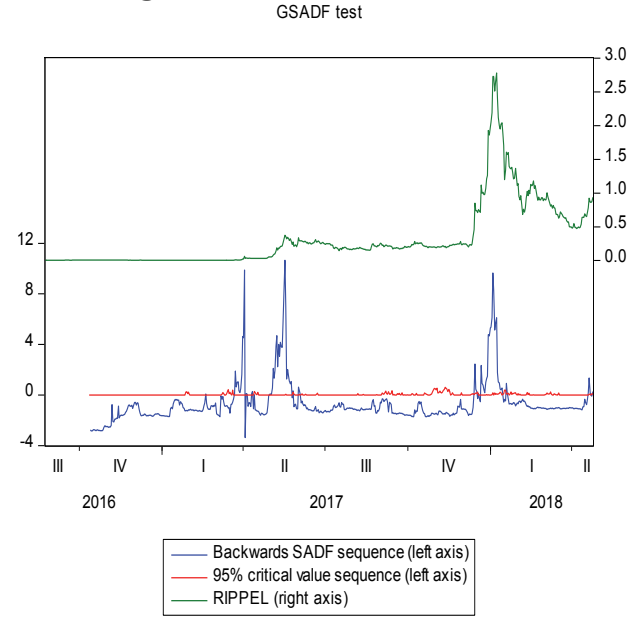

Fig. 3. GSADF test for Ripple

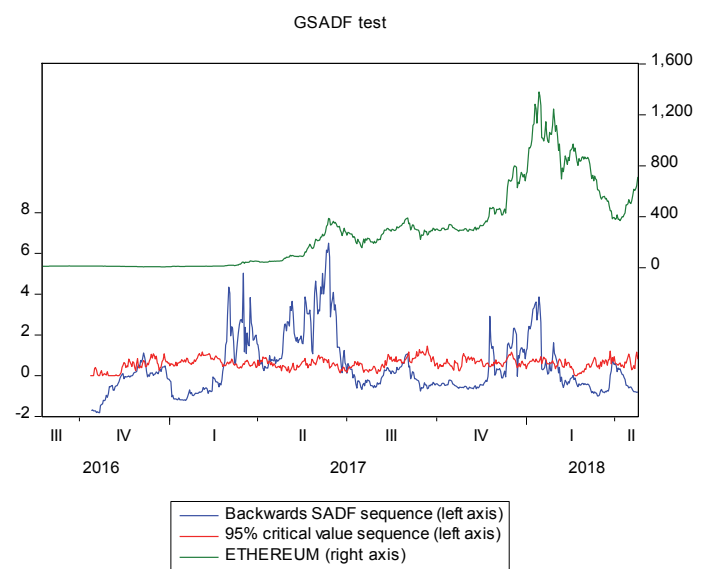

Fig. 2. GSADF test for Ethereum

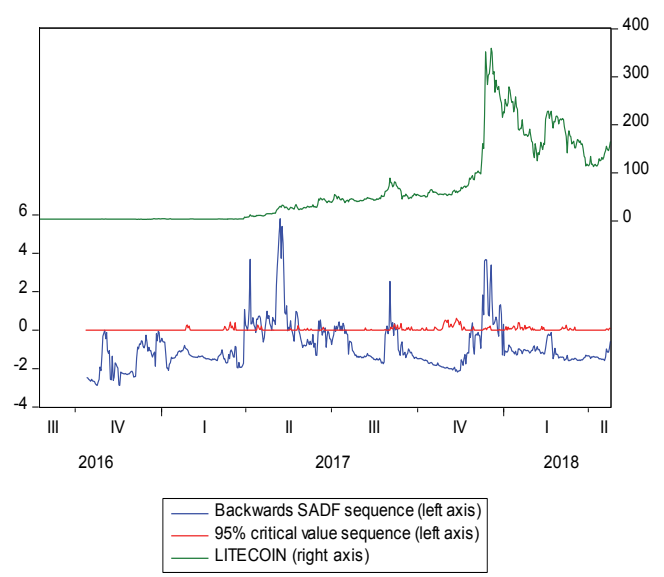

Fig. 4. GSADF test for Litecoin

An estimated t-stat of 5.804 was derived from Litcoin which is by far more than $1 \%$ critical value of 1.791 explosive alternatives. Thus, it shows that Litecoin possesses price explosive characteristics as shown by the GSADF Date-stamping bubble diagram which ranges from; (2017Q1 to 2017Q2), (2017 to 2017Q3), and from (2017Q4 to 2018Q1). Finally, the Ripple, with t-stat of 10.66 which is greater than the $1 \%$ critical value of the eruptive alternative of 1.791 . This denotes that Ripple prices rejected 
the null hypothesis of a random walk, showing a significant level of price bubble in the series and this bubble phases are been captured in the GSADF Date-stamping result. This bubble periods ranges from the (2017Q1 to 2017Q2), and between the 4th quarter of 2017 to the 1 st quarter of 2018.

\subsection{Power Spectrum Interpretation}

As explained earlier in the methodology section, the power spectrum shows the areas of high and low amplitude and also the magnitude of the volatility in a particular region in the data set. Below are the power spectra for crypto-currencies employed in this study.

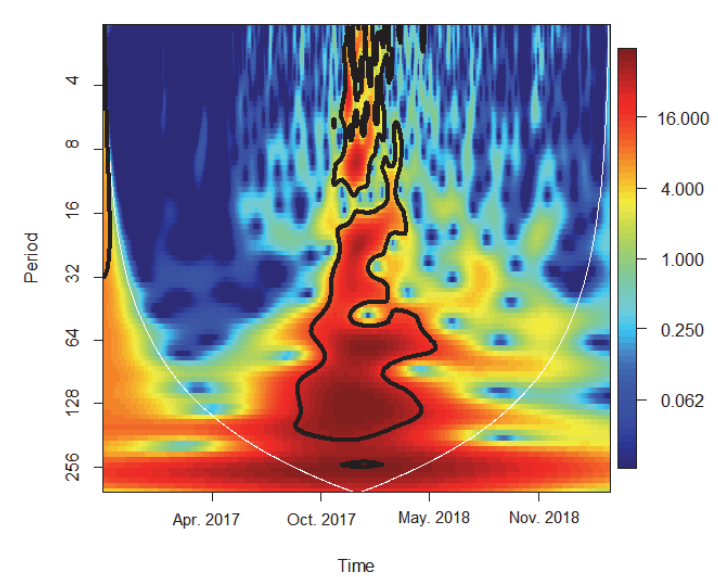

Fig. 5. Power Spectrum for Bitcoin

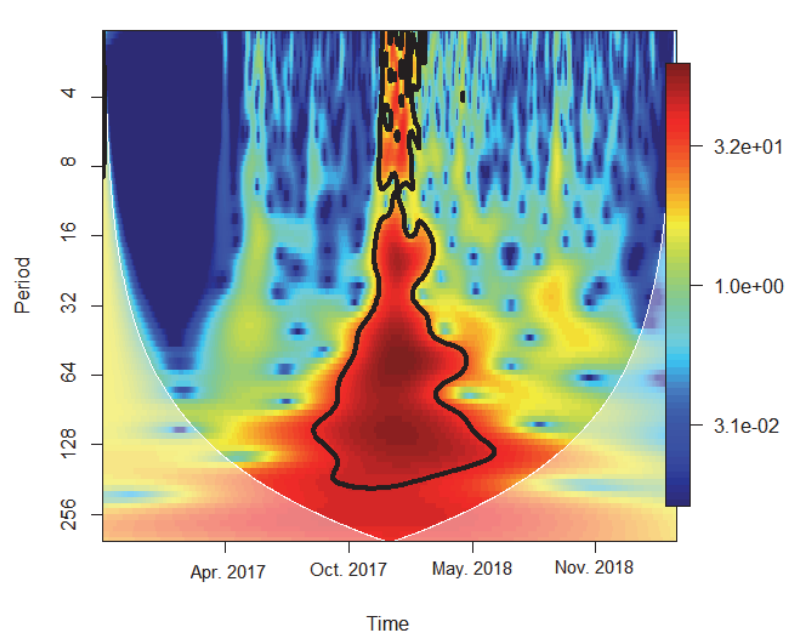

Fig. 7. Power Spectrum for Ripple

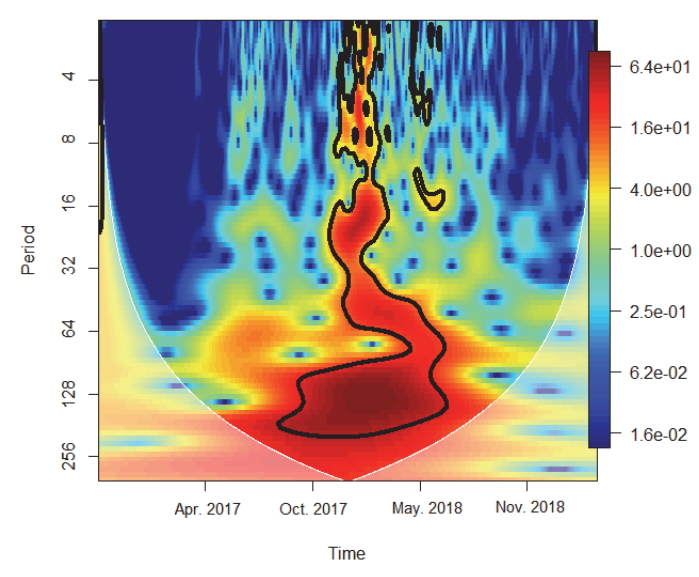

Fig. 6. Power Spectrum for Etherium

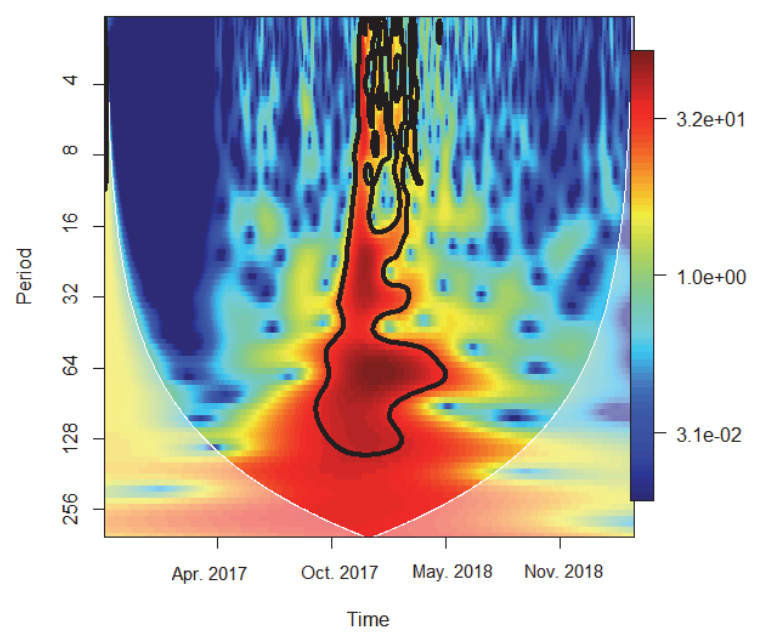

Fig. 8. Power Spectrum for Litecoin

Based on the continual transformation of wavelet, the above Figs. 5, 6, 7, and 8 demonstrate the variations in the raw data for Bitcoin, Etherium, Litecoin, and Ripple. At the middle of dataset period, the red area on the left (right side) of the periodic power spectra figures suggest that there is a considerable variation, while the bottom (top) red area is a high vulnerability at low (high) frequencies. A high variation in Bitcoin surfaced as vividly indicated by Fig. 5 from the 3rd quarter of 2017 to 1 st quarter of 2018. Furthermore, high variation in Etherium surfaced as clearly indicated by Fig. 6 from 4th quarter of 2017 to 2nd quarter of 2018, in the case of Ripple, a high variation shows up as vividly indicated by Fig. 7 from 3rd quarter of 2017 to 1st quarter of 2018, and finally, pertaining to Litecoin, a high variation surfaced as vividly indicated by Fig. 8 from 3rd quarter of 2017 to 2nd quarter of 2018.

Fig. 9, Fig. 10, and Fig. 11 indicate wavelet coherence scalogram (Bitcoin, Etherium, Litecoin, and Ripple. The wavelet coherence software initiated by Grinsted et al. (2004) was employed in this study. Time is shown by the horizontal axis; relationship between 2 variables that occurred in the earlier part of the series are captured at the leftmost area of the wavelet diagram while relationship that occurred towards the end of the series are at the rightward areas of the wavelet diagram. The vertical axis holds information regarding the time periods; the lower period bands (greater frequencies) are reflected close to 
the top and the lower frequencies (higher bands) close to the bottom. The wavelets coherence indicates the regions of the timefrequency space in which the two series move simultaneously. In the inner sphere of the time-frequency space, the warmer the color, the higher the coherence (that can be construed as correlation); the colors used on this work, range from dark blue ( 0 , indicates coherence does not surface) to dark red (1, reflects strong correlation). A thick black line surrounds statistically significant regions of coherence. Two things appear in the direction of the arrows: the correlation and the time series at that point leading the relationship. Arrows pointing leftwards reflects anti-phase, implying that there is negative relationship between the two-time series while arrows pointing rightwards indicates in-phase which implies that there is positive relationship between the two-time series. On the other hand, a downward pointing arrow suggests the first series leads the second which interpret as; prices behaviors in the first series causes subsequent price behaviors in the second series, vice versa.

Although, the time series perceived are finite, not every data is necessary for the areas at the beginning and end of the data (particularly for the higher periods). One option selected is to pad the data set with nulls were necessary to make computing viable. Null padding will, however, affect the results reliability. This distinction in the reliability of results should be depicted by using a cone of influence. The pale colors portray those regions with little reliable results outside the cone of influence. More data are required in higher bands to calculate the cone structure. Figs. 9, 10 and 11 show the scalograms of the wavelet coherence between Bitcoin and varying combinations of other crypto-currencies (Etherium, Ripple, and Litecoin). The scalograms for all relationships are estimated separately. A view of a series enables the comparison with different crypto-currencies of any associations with Bitcoin. Staring down, a column shows how Bitcoin is correlated with a certain crypto-currency. The shaded red areas suggest places within the pricing series of crypto-currencies recognized as bubble-like structures utilizing the GSADF bubble test discussed earlier. However, it should be observed that for Bitcoin, Etherium, Ripple and Litecoin between 2016 and 2018 the dark blue areas were due to a shortage of data for these metrics before 2016. In order to be clear, it is necessary to define the short, medium and long term explicitly. Throughout this work, 2-4 and 4-8 days' period band are referred to short term. The medium-term refers to 8-16, and 16-32 days' period band. 32-64, and 64-256-days period bands are referred to as the long-term.

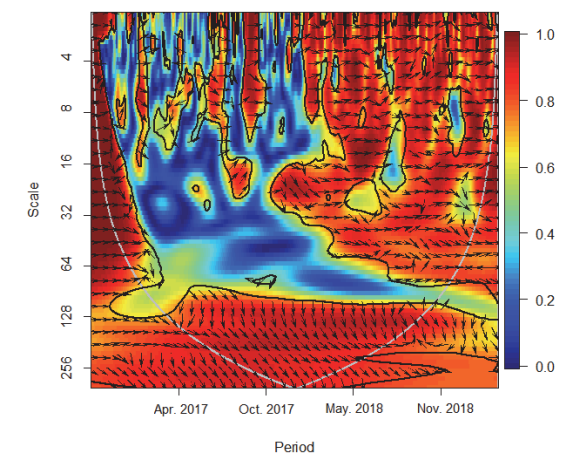

Fig. 9. Wavelet Coherence between Bitcoin and Ethereum

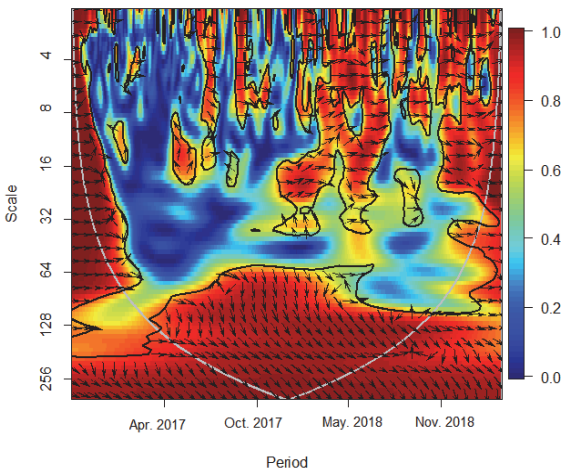

Fig. 10. Wavelet Coherence between Bitcoin and Ripple

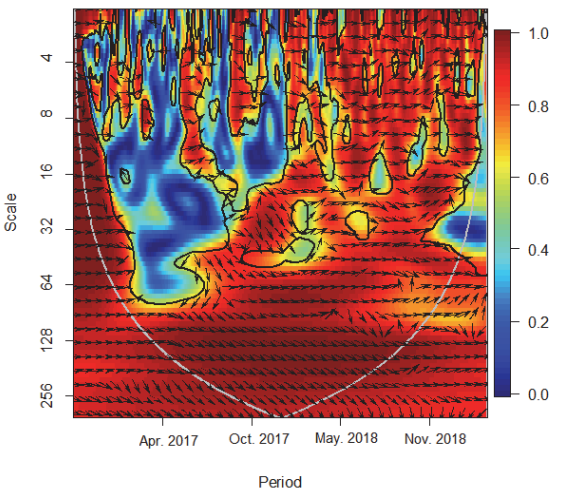

Fig. 11. Wavelet Coherence between Bitcoin and Litecoin

To capture the time frequency dependency of Bitcoin and other cryptocurrencies, wavelet coherence test is used and the outcomes of the test are reported in Figs (9-11). As clearly seen in Fig. 9, since the scale of 4 and 8 periods indicates short-run and the way of the majority of arrows are to the right, there is positive correlation between Bitcoin prices and Etherium prices. However, the direction of the arrows turns into down with a higher level of warmness in Fig. 9 meaning that Bitcoin lead Etherium in the long run. This result shows that price behaviors of Etherium can be explained by Bitcoin price trends. It is clear from the Fig. 10 above that Bitcoin lead Rippel in the short run, medium run, and the long run. A positive relationship can be derived from this relationship as depicted by the rightward pointing arrows. Furthermore, the scalogram above also shows a strong relationship in the long-run between Bitcoin, and Rippel than in the short term, and medium term by reflecting a higher level of warmness, also indicating how the price of Bitcoin is an important predictor of the price of Rippel in the long-run. The Fig. 11 illustrates that Bitcoin are correlated with Litecoin in the short run, with a corresponding positive relationship. In addition, the figure also shows a higher level of correlation in the long-run when compared to the short-run, and medium run between Bitcoin and Litecoin as seen with a higher level of warmness in the color variation. In the long run, the outcomes of the wavelet coherence test shows that the price of Bitcoin is the main driver of the price of Litecoin in the long-run.

\section{Conclusion}

The study aims (i) to explore the time frequency dependency of the price of Bitcoin and the price of other popular cryptocurrencies and (ii) to find out whether the crypto-currencies used contained a evidence of a price bubble(s). Therefore, we used 
wavelet coherence and GSADF approaches in the present study. The outcome reveals the existence of bubbles among all the variables considered in the study. The outcomes from the wavelet coherence analysis further shed more light into the relationship between Bitcoin and its counterparts. The main finding reflects that there exists a short term, medium term, and long-term relationships among the crypto-currencies. Based on the results of the GSADF test and further analysis, some explanations are provided based on the strong long-term relationship between the variables.

These findings are useful for anyone who explores Bitcoin's dependency on other crypto-currencies in academia or industry. The reinforcement of bubble relationship also shows how crypto-currencies can be utilized as speculative assets. Coherence strengthening is far less prominent in the short and medium term in bubble regimes. In the short run, daily news items and intraday trading activities can hide the consequences of bubbles. Furthermore, the relationship between Bitcoin and its counterparts (Etherium, Ripples, and Litecoin) is also shown to be inconsistent and generally weak in the short and mediumterm. However, there is proof that Bitcoin will lead in the medium and long term when it comes to compare with the other crypto-currencies.

With regards to the links between different crypto-currencies, significant correlation between these coins is noted in the short term, due to the similar financial characteristics they possess. It is shown that short-term correlations between crypto-currencies are again based on news items and broad events on the market. Substantially, the results outlined here are expected to stimulate further work in this field, particularly in relation to bubble dynamics in crypto-currency markets, and how changes in Bitcoin price affect them over time. In addition, a short-term relationship between crypto-currencies and speculative activities might warrant research on mechanisms that influence crypto-currency markets in the events such as news items possibly in the same way as for other asset classes, and might lead to a portfolio-balancing strategy to adapt automatically to the market news.

\section{References}

Astill, S., Harvey, D. I., Leybourne, S. J., \& Taylor, A. R. (2017). Tests for an end-of-sample bubble in financial time series. Econometric Reviews, 36(6-9), 651-666.

Bariviera, A. F., Basgall, M. J., Hasperué, W., \& Naiouf, M. (2017). Some stylized facts of the Bitcoin market. Physica A: Statistical Mechanics and its Applications, 484, 82-90.

Baur, D. G., Dimpfl, T., \& Kuck, K. (2018). Bitcoin, gold and the US dollar-A replication and extension. Finance Research Letters, 25, 103-110.

Bouri, E., Gupta, R., Lahiani, A., \& Shahbaz, M. (2018). Testing for asymmetric nonlinear short-and long-run relationships between bitcoin, aggregate commodity and gold prices. Resources Policy, 57, 224-235.

Cap, C. M. (2018). Cryptocurrency market capitalizations. Retrieved on January, 21, 2018.

Chueng, A., Roca, E., \& Su, J. J. (2015). Crypto-currency bubbles: an application of the Phillips-Shi-Yu (2013) methodology on Mt. Gox bitcoin prices. Applied Economics, 47(23), 2348-2358.

Corbet, S., McHugh, G., \& Meegan, A. (2017). The influence of central bank monetary policy announcements on cryptocurrency return volatility. Investment Management \& Financial Innovations, 14(4), 60.

Dickey, D. A., \& Fuller, W. A. (1979). Distribution of the estimators for autoregressive time series with a unit root. Journal of the American Statistical Association, 74(366a), 427-431.

Dyhrberg, A. H. (2016). Bitcoin, gold and the dollar-A GARCH volatility analysis. Finance Research Letters, 16, 85-92.

Fantazzini, D. (2016). The oil price crash in 2014/15: Was there a (negative) financial bubble? Energy Policy, 96, 383-396.

Fry, J., \& Cheah, E. T. (2016). Negative bubbles and shocks in cryptocurrency markets. International Review of Financial Analysis, 47, 343-352.

Goupillaud, P., Grossmann, A., \& Morlet, J. (1984). Cycle-octave and related transforms in seismic signal analysis. Geoexploration, 23(1), 85-102.

Grinsted, A., Moore, J. C., \& Jevrejeva, S. (2004). Application of the cross wavelet transform and wavelet coherence to geophysical time series. Nonlinear Processes in Geophysics, 11(5/6), 561-566.

Kristoufek, L. (2013). BitCoin meets Google Trends and Wikipedia: Quantifying the relationship between phenomena of the Internet era. Scientific reports, 3, 3415.

Larsen, E. S. (1997). Theories and tests for bubbles (Master's thesis, Universitetet i Tromsø).

Osterrieder, J., \& Lorenz, J. (2017). A statistical risk assessment of Bitcoin and its extreme tail behavior. Annals of Financial Economics, 12(01), 1750003.

Pal, D., \& Mitra, S. K. (2017). Time-frequency contained co-movement of crude oil and world food prices: A wavelet-based analysis. Energy Economics, 62, 230-239.

Phillips, P.C.B., Shi, S., \& Yu, J. (2012) Testing for multiple bubbles. Cowles Foundation Discussion Paper No.1843

Phillips, P. C., Wu, Y., \& Yu, J. (2011). Explosive behavior in the 1990s Nasdaq: When did exuberance escalate asset values?. International Economic Review, 52(1), 201-226.

Phillips, P. C., \& Yu, J. (2011). Dating the timeline of financial bubbles during the subprime crisis. Quantitative Economics, 2(3), 455-491. 
Phillips, P. C., Shi, S., \& Yu, J. (2015). Testing for multiple bubbles: Historical episodes of exuberance and collapse in the S\&P 500. International Economic Review, 56(4), 1043-1078.

Phillips, P. C., Wu, Y., \& Yu, J. (2011). Explosive behavior in the 1990s Nasdaq: When did exuberance escalate asset values?. International Economic Review, 52(1), 201-226.

Pichl, L., \& Kaizoji, T. (2017). Volatility analysis of bitcoin. Quantitative Finance and Economics, 1, 474-485.

Salhi, B., \& Alflayyeh, S. (2016). Impact of Speculation and Bubble Detection in Stock Markets: The Tunisian and the Moroccan Cases. Journal of Management and Strategy, 7(2), 73-89.

Semiromi, B., \& Reza, M. (2010). Literatures about asset price bubbles and monetary policies. In International Conference on Applied Economics, ICOAE (Vol. 2010, pp. 695-703).

Su, C. W., Li, Z. Z., Tao, R., \& Si, D. K. (2018). Testing for multiple bubbles in bitcoin markets: A generalized sup ADF test. Japan and the World Economy, 46, 56-63.

Urquhart, A., \& Zhang, H. (2018). Is Bitcoin a hedge or safe-haven for currencies. An intraday analysis, 1-25.

Torrence, C., \& Compo, G. P. (1998). A practical guide to wavelet analysis. Bulletin of the American Meteorological society, 79(1), 61-78.

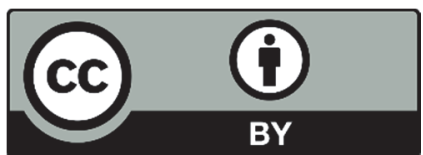

(C) 2019 by the authors; licensee Growing Science, Canada. This is an open access article distributed under the terms and conditions of the Creative Commons Attribution (CC-BY) license (http://creativecommons.org/licenses/by/4.0/). 